\title{
As principais causas de morte maternas entre mulheres no Brasil
}

\author{
The main causes of maternal death among women in Brazil
}

Las principales causas de muerte materna entre las mujeres del Brasil

Elaine da Silva Costa ${ }^{1 *}$, Rosemary Baima de Oliveira ${ }^{1}$, Graciana de Sousa Lopes².

\section{RESUMO}

Objetivo: Analisar as principais causas de mortalidade materna entre mulheres no Brasil. Métodos: Trata-se de uma pesquisa descritiva, exploratória e qualitativa, que seguirá como base de fundamentação teórica, periódicos de repositórios virtuais. Os artigos completos utilizados neste trabalho serão em português, inglês e disponibilizados gratuitamente por meio digital, publicados na última década. Resultados: Os artigos que serviram de base para discussão dos resultados de acordo com os objetivos dos do trabalho, demonstraram que no Brasil $80 \%$ dos óbitos maternos são decorrentes de causas obstétricas diretas, e, por outro lado, $20 \%$ são responsáveis por causas obstétricas indiretas. No contexto brasileiro, as causas hipertensivas estão no topo da lista, seguida das hemorrágicas e infecções. O óbito materno tornou-se um dos grandes desafios da saúde pública no Brasil. Considerações finais: Para reduzir o índice de óbito materno no âmbito hospitalar, algumas condutas são necessárias, o papel do profissional de enfermagem obstétrica na prevenção da mortalidade materna e o aprimoramento no diagnóstico precoce no tratamento relacionado ao período gravídico puerperal.

Palavras-chave: Mortalidade materna, Causas de óbitos, Condutas de prevenção.

\begin{abstract}
Objective: To analyze the main causes of maternal mortality among women in Brazil. Methods: This is a descriptive, exploratory and qualitative research, which will follow as a theoretical basis, periodicals of virtual repositories. The complete articles used in this work will be in Portuguese, English and made available free of charge through digital means, published in the last decade. Results: The articles that served as a basis for discussion of the results according to the objectives of the work, demonstrated that in Brazil $80 \%$ of maternal deaths are due to direct obstetric causes, and, on the other hand, $20 \%$ are responsible for indirect obstetric causes. In the Brazilian context, hypertensive causes are at the top of the list, followed by hemorrhagic and infections. Maternal death has become one of the great public health challenges in Brazil. Final considerations: To reduce the rate of maternal death in the hospital environment, some behaviors are necessary, the role of the obstetric nursing professional in the prevention of maternal mortality and the improvement in the early diagnosis in the treatment related to the puerperal gravidic period.
\end{abstract}

Keywords: Maternal mortality, Causes of deaths, Prevention conducts.

\section{RESUMEN}

Objetivo: Analizar las principales causas de la mortalidad materna de las mujeres en Brasil. Métodos: Se trata de una investigación descriptiva, exploratoria y cualitativa, que seguirá como base teórica, las publicaciones periódicas de los depósitos virtuales. Los artículos completos utilizados en este trabajo estarán

${ }^{1}$ Centro Universitário Fametro (CEUNI-FAMETRO), Manaus - AM. *E-mail: elainesilvacostaflor@gmail.com

2Universidade Federal do Amazonas (UFAM), Manaus - AM. 
en portugués, inglés y disponibles gratuitamente por medios digitales, publicados en la última década. Resultados: Los artículos que sirvieron de base para la discusión de los resultados de acuerdo con los objetivos del trabajo, demostraron que en Brasil el $80 \%$ de las muertes maternas se deben a causas obstétricas directas y, por otro lado, el $20 \%$ son responsables de causas obstétricas indirectas. En el contexto brasileño, las causas de la hipertensión son las primeras de la lista, seguidas por las causas hemorrágicas y las infecciones. La muerte materna se ha convertido en uno de los principales desafíos de la salud pública en el Brasil. Consideraciones finales: Para reducir la tasa de mortalidad materna en el ámbito hospitalario, son necesarios algunos comportamientos, el papel del profesional de la enfermería obstétrica en la prevención de la mortalidad materna y la mejora del diagnóstico precoz en el tratamiento relacionado con el período gravitatorio puerperal.

Palabras clave: Mortalidad materna, Causas de muerte, Conductas de prevención.

\section{INTRODUÇÃO}

$\mathrm{Na}$ atualidade, os levantamentos sobre morte materna no Brasil vêm se reduzindo devido à realização de ações de prevenção na diminuição dos níveis de óbito materno. De acordo com o Sistema de Informações sobre Mortalidade (SIM), no período de 2004 a 2014 no Brasil ocorreu 18.364 óbitos maternos, estando a região Nordeste com o maior quantitativo de óbitos, cerca de 6.514 que correspondente a $35,47 \%$ da mortalidade materna do país, a menor taxa nacional ficou com a região Centro-Oeste, com um total de 1.422 óbitos, em torno de $7,74 \%$, apesar dos esforços desempenhados muitas mulheres ainda vêm à óbito durante a gestação, por volta de $90 \%$ dos óbitos são causas evitáveis (VIANA RC, et al., 2011; DATASUS, 2020).

O Brasil na tentativa de minimizar as desigualdades e aprimorar o Índice de Desenvolvimento Humano (IDH), vêm assumindo oito iniciativas reconhecidas como Objetivos de Desenvolvimento do Milênio, dentre elas a redução da mortalidade materna. Conforme a Organização Mundial de Saúde (OMS) o país está entre os 11 países latino-americanos que alcançaram aumentos significativos na redução de óbitos associados à gravidez ou parto no período de 1990 a 2015 (GUIMARÃES TA, et al., 2017).

A Política Nacional de Atenção Integral à Saúde da Mulher (PNAISM), do Ministério da Saúde (MS) juntamente com o Sistema Único de Saúde (SUS) vêm seguindo os princípios de igualdade e universalidade na redução da mortalidade materna com atenção integral à saúde da mulher (FERREIRA LO, 2013).

De acordo a OMS, a morte de mulheres no período de gestação ou após 42 dias do término da gravidez, é considerado como mortalidade materna (BRASIL, 2006). Na literatura as principais causas obstétricas diretas de mortalidade materna estão relacionadas às complicações na gestação ou no parto, em virtude de tratamento inapropriado, técnicas incorretas e omissões. Dentre as causas indiretas, estão as que se originam de doenças existentes antes da gestação ou de uma patologia desenvolvida durante a gravidez (FERRAZ L e BORDIGNON M, 2012). A assistência irregular à gestante pode resultar em uma morte materna que poderia ter sido evitada por intermédio de desenvolvimento de ações de qualidade (SANTOS DR, et al., 2017).

A OMS calcula o coeficiente de mortalidade materna pelo número de óbitos maternos a cada 100 mil nascidos vivos, sendo aceitável o limite de 20 mortes a cada 100 mil nascidos vivos. Esse coeficiente de mortalidade com um número de óbitos entre 20 e 49 é considerado um valor médio, e de 50 a 149 óbitos, um valor alto e muito alto quando o valor é superior a 150 óbitos por 100 mil nascidos vivos (MEDEIROS LT, 2018).

De acordo com MS brasileiro, os dados de 1990 a 2020 foi evidenciado uma redução na mortalidade materna, caindo de 141 para 68 óbitos por 100 mil nascidos vivos, representando uma baixa de $51 \%$, mesmo com a queda no número de óbitos, esse coeficiente está acima do limite aceitável pela OMS (SERRUYA SJ, et al., 2004).

Devido à problemática, surgiu a necessidade do desenvolvimento de um estudo na contribuição de pesquisas sobre o problema de mortalidade materna. Este estudo é fundamental para a identificação de grupos populacionais mais vulneráveis a óbitos na gestação, e ainda no reconhecimento das causas dessas 
mortes, buscando o desenvolvimento de políticas públicas e de ações na diminuição do número de casos. 0 presente trabalho tem como objetivo realizar um levantamento bibliográfico sobre quais são as principais causas que levam a mortalidade materna entre mulheres em idade fértil no Brasil.

\section{MÉTODOS}

Trata-se de um estudo exploratório, descritivo, do tipo Revisão Integrativa da Literatura (RIL). Para coleta de dados, foram utilizadas as bibliotecas virtuais de pesquisa: Biblioteca Científica Eletrônica Online (SCIELO) e Literatura Latino Americana e do Caribe em Ciências da Saúde (LILACS) mediantes os seguintes descritores: "Maternal Mortality in Brazil", marcando na busca principal: "Mortalidade materna", "Causas de Morte materna" e Mortalidade.

Como critérios de elegibilidade foram selecionados artigos originais, disponibilizados gratuitamente, em língua portuguesa e língua inglesa, publicados no período de 2010 a 2020, que tratam do tema pesquisado. Critérios de inelegibilidade foram: artigos com texto incompleto, resumos, monografias, dissertações de mestrado, teses de doutorado.

Os artigos foram selecionados de acordo com os critérios de elegibilidade, inelegibilidade, e posteriormente foi realizada a análise de resumos e finalmente os artigos foram lidos na integra, sendo elaborado um instrumento para a coleta de informações direto das bases de dados.

\section{RESULTADOS E DISCUSSÃO}

A busca dos artigos foi realizada por meio da Biblioteca Virtual em Saúde (BVS), com busca na Base de dados da Medical Literature Analysis and Retrieval System Online (MEDLINE), Base de Dados da Enfermagem (BDENF) Literatura Latino-Americana e do Caribe em Ciências da Saúde (LILACS).

A busca dos estudos ocorreu no mês de setembro de 2020, com o descritor, "Maternal Mortality in Brazil" com a livre tradução "Mortalidade Materna no Brasil", indexado na Biblioteca Virtual da Saúde (BVS). Utilizando o período temporal dos últimos dez anos (2010 a 2020), no intuito de propiciar a exploração da literatura científica atual, totalizando 131 artigos (BDENF 10), (MEDLINE 65), (LILACS 56). Utilizando o filtro para o idioma, observou-se (Português 76) e (Inglês 55).

Conforme descrito anteriormente, foram selecionados alguns dos artigos científicos nacionais e internacionais, que se encontram no (Quadro 1), estes artigos servirão de base para discutir e argumentar, os resultados de acordo com o tema e os objetivos desse trabalho. 
Quadro 1 - Artigos selecionados para composição dos resultados e discussão.

\begin{tabular}{|c|c|c|c|c|c|c|}
\hline ID & Título & Autor & Ano & Idiomas & Plataforma & Resultados \\
\hline 1 & $\begin{array}{l}\text { Mortalidade materna: comparativo } \\
\text { após implantação da Rede Mãe } \\
\text { Paranaense }\end{array}$ & $\begin{array}{c}\text { SCHOLZE AR, } \\
\text { et al., }\end{array}$ & 2020 & Port & J. nurs. health & $\begin{array}{l}\text { O estudo mostrou uma queda na taxa de mortalidade para } \\
\text { as mulheres com menos de oito anos de estudo, sem } \\
\text { companheiro e com extremos de idade (menos de } 20 \text { anos } \\
\text { e mais de } 34 \text { anos). }\end{array}$ \\
\hline 2 & Ocupação e mortalidade materna & $\begin{array}{l}\text { ASSIS AIF e } \\
\text { SANTANA VS }\end{array}$ & 2020 & Port & $\begin{array}{l}\text { Revista de Saúde } \\
\text { Pública - SCIELO }\end{array}$ & $\begin{array}{l}\text { Os achados deste estudo podem contribuir para uma melhor } \\
\text { visibilidade do problema e alertar para novos aportes } \\
\text { voltados para grupos ocupacionais mais vulneráveis. }\end{array}$ \\
\hline 3 & $\begin{array}{l}\text { Perfil da Mortalidade Materna: } \\
\text { Uma Revisão Integrativa da } \\
\text { Literatura }\end{array}$ & $\begin{array}{c}\text { SCARTIN J, et } \\
\text { al., }\end{array}$ & 2019 & Port & $\begin{array}{l}\text { J. res.: fundam. } \\
\text { care. online }\end{array}$ & $\begin{array}{l}\text { O perfil epidemiológico dos óbitos maternos é influenciado } \\
\text { por fatores sociais, os quais refletem as desigualdades que } \\
\text { assolam o mundo, a disparidade nas formas de acesso aos } \\
\text { serviços de saúde, a educação e demais fatores que } \\
\text { repercutem num grupo vulnerável para índices alarmantes. }\end{array}$ \\
\hline 4 & $\begin{array}{l}\text { Actions of primary health care } \\
\text { professionals } \\
\text { to reduce maternal mortality in the } \\
\text { Brazilian Northeast }\end{array}$ & $\begin{array}{l}\text { FIGUEIREDO } \\
\text { KMS, et al., }\end{array}$ & 2018 & Inglês & $\begin{array}{c}\text { Port of Springer } \\
\text { Nature }\end{array}$ & $\begin{array}{l}\text { O estudo mostra que a interdisciplinaridade entre os } \\
\text { profissionais da APS facilita o atendimento integrado à } \\
\text { gestante. }\end{array}$ \\
\hline 5 & $\begin{array}{l}\text { Maternal Mortality in Brazil: } \\
\text { Proposals and Strategies for its } \\
\text { Reduction }\end{array}$ & $\begin{array}{l}\text { PACAGNELLA } \\
\text { RC, et al., }\end{array}$ & 2018 & Inglês & $\begin{array}{l}\text { Revista de Saúde } \\
\text { Pública - SCIELO }\end{array}$ & $\begin{array}{l}\text { A morte materna não pode ser atribuída a um único } \\
\text { fator; portanto, ações devem ser formuladas de forma } \\
\text { sistemática para possibilitar a redução dessas altas taxas de } \\
\text { mortalidade materna. }\end{array}$ \\
\hline 6 & $\begin{array}{l}\text { Strategy for Zero Maternal Deaths } \\
\text { by Hemorrhage in Brazil: } \\
\text { A Multidisciplinary Initiative to } \\
\text { Combat Maternal Morbimortality }\end{array}$ & $\begin{array}{l}\text { OSANAN GC, } \\
\text { et al., }\end{array}$ & 2018 & Inglês & $\begin{array}{l}\text { Revista de Saúde } \\
\text { Pública - SCIELO }\end{array}$ & $\begin{array}{l}\text { A principal missão do zero morte materna por hemorragia é } \\
\text { contribuir para a melhoria da qualidade da assistência } \\
\text { obstétrica no Brasil, e conscientizar que todas as pessoas, } \\
\text { nos cargos ou funções que já ocupam na sociedade, são } \\
\text { responsáveis pela prevenção da morte materna. }\end{array}$ \\
\hline
\end{tabular}




\begin{tabular}{|c|c|c|c|c|c|c|}
\hline ID & Título & Autor & Ano & Idiomas & Plataforma & Resultados \\
\hline 7 & $\begin{array}{l}\text { Parto cesário e parto normal: Uma } \\
\text { abordagem acerca de riscos e } \\
\text { benefícios }\end{array}$ & $\begin{array}{l}\text { VICENTE AC, } \\
\text { et al., }\end{array}$ & 2017 & Pt-br & $\begin{array}{c}\text { Temas em Saúde } \\
\text { - FIOCRU }\end{array}$ & $\begin{array}{l}\text { O estudo mostra que a realidade das gestantes nos dias } \\
\text { atuais ainda persiste em grandes números o parto casario, } \\
\text { mesmo com campanhas educativas proporcionadas pelas } \\
\text { políticas de saúde, seja por profissionais de saúde, } \\
\text { propagandas televisivas, entre outros meios de divulgações. }\end{array}$ \\
\hline 8 & $\begin{array}{l}\text { Óbito materno, causalidade e } \\
\text { estratégias de vigilância: uma } \\
\text { revisão integrativa }\end{array}$ & $\begin{array}{l}\text { SOARES FAF, } \\
\text { et al., }\end{array}$ & 2017 & Port & $\begin{array}{l}\text { Revista Eletrônica } \\
\text { Acervo Saúde }\end{array}$ & $\begin{array}{l}\text { Apesar de terem causas variadas, tais óbitos se dão por } \\
\text { causas evitáveis, como as doenças hipertensivas e } \\
\text { hemorrágicas da gestação, o que não justifica tais taxas. }\end{array}$ \\
\hline 9 & $\begin{array}{l}\text { Estudo da mortalidade materna na } \\
\text { Região do Alto Jequitinhonha, } \\
\text { Minas Gerais }\end{array}$ & $\begin{array}{l}\text { DIAS JA, et } \\
\text { al., }\end{array}$ & 2016 & Port & $\begin{array}{l}\text { Revista de Saúde } \\
\text { Pública - SCIELO }\end{array}$ & $\begin{array}{l}\text { Para redução de mortes materna, devem ser propostas } \\
\text { medidas de prevenção, planejamento familiar abrangente, } \\
\text { assistência pré-natal adequada, equipe qualificada para } \\
\text { atendimento e vigilância no período puerperal. }\end{array}$ \\
\hline 10 & Mortalidade materna & $\begin{array}{l}\text { GONÇALVES } \\
\text { DJM, et al., }\end{array}$ & 2015 & Port & $\begin{array}{l}\text { Revista de Saúde } \\
\text { Pública - SCIELO }\end{array}$ & $\begin{array}{l}\text { A mortalidade pode ser evitada com programas clínico- } \\
\text { educacionais que não necessitam de grandes tecnologias, } \\
\text { como: o } \\
\text { planejamento familiar, a vinculação do pré-natal ao parto e } \\
\text { educação sexual. }\end{array}$ \\
\hline 11 & $\begin{array}{l}\text { Assistência pré-natal por } \\
\text { profissionais de enfermagem no } \\
\text { município de rio } \\
\text { Branco, Acre, Amazônia }\end{array}$ & $\begin{array}{l}\text { CUNHA MA, } \\
\text { et al., }\end{array}$ & 2012 & Port & $\begin{array}{l}\text { Revista Baiana } \\
\text { de Saúde Pública } \\
\text { - SCIELO }\end{array}$ & $\begin{array}{l}\text { Os resultados deste estudo ofereceram oportunidade para } \\
\text { se refletir sobre a qualidade da atenção pré-natal nos } \\
\text { serviços estudados e a relevância do trabalho realizado } \\
\text { pelos enfermeiros, para o município e para as gestantes em } \\
\text { particular. }\end{array}$ \\
\hline 12 & $\begin{array}{l}\text { Características epidemiológicas } \\
\text { dos óbitos maternos ocorridos em } \\
\text { Recife, PE, Brasil (2000-2006) }\end{array}$ & $\begin{array}{l}\text { CORREIA RA, } \\
\text { et al., }\end{array}$ & 2011 & Port & $\begin{array}{l}\text { Revista Brasileira } \\
\text { de Enfermagem - } \\
\text { SCIELO }\end{array}$ & $\begin{array}{l}\text { Este estudo descreve as características epidemiológicas } \\
\text { dos óbitos. Os dados obstétricos mostram que os } \\
\text { transtornos hipertensivos constituem a principal causa de } \\
\text { óbito. }\end{array}$ \\
\hline
\end{tabular}

Fonte: Costa ES, et al., 2020. 
Após a análise dos achados, escolheu-se três categorias, para melhor compreensão do assunto e alcance do objetivo deste estudo.

\section{Categoria I: Política e programas voltados para a saúde integral da mulher}

No ano de 2000 a Organização das Nações Unidas (ONU) constituída por 192 países-membros assinaram um pacto com 8 objetivos milênio. Um conjunto de medidas e metas elaboradas para desenvolvimento da sociedade até $\mathrm{o}$ ano de 2015. O 5o objetivo tem como finalidade melhorar a saúde das gestantes e a diminuição da mortalidade materna para 35 óbitos por 100 mil nascidos vivos em 2015. O Brasil não alcançou essa estimativa, tendo como resultado 62 mortes por óbitos maternos. Os países-membros da UNO assinaram um novo pacto para os anos de 2015 a 2030, com os 17 objetivos para o desenvolvimento sustentável, estando a mortalidade materna no $3^{\circ}$ objetivo, tendo como meta estipulada pelo Ministério da Saúde, 30 óbitos por 100 mil nascidos vivos até 2030 (BRASIL, 2018).

Nos anos de 2000 a 2016 observou que o cenário de mortalidade materna no Brasil teve uma decadência não linear atingindo a maior taxa em 2006, cerca de $77,2 \%$ e a menor taxa em 2012, em torno de 59,3\% na taxa de mortalidade materna. No período de 2012 até 2016, os índices são 59,3\%, 62,1\%, 63,8\%, 62,0\% e $64,4 \%$, a taxa de mortalidade materna no país teve um crescimento que atrapalha o alcance do acordo firmado para 2030. No cenário por regiões brasileiras no ano de 2016, observou se as regiões com os maiores índices, situando-se a região norte com $84,5 \%$, seguido do Nordeste com $70 \%$ e o Centro-Oeste com $67,3 \%$. (BRASIL, 2018; MARINHO F, 2018).

O constante avanço na assistência à gravidez e ao período neonatal apresentaram respostas positivas para a saúde perinatal. No entanto, evidenciam as desigualdades na acessibilidade da assistência à saúde, sendo uma das causas de risco que colaboram no índice de mortalidade materna (KALE PL, et al., 2018). Nas décadas de 30 a 70, os programas materno-infantis eram submetidos com uma visão reducionista da mulher relacionada a condição biológica e o papel social da mulher (BRASIL, 2012).

No ano de 1984, o MS criou o Programa de Assistência Integral à Saúde da Mulher (PAISM), apresentando ações educativas, medidas de diagnóstico, tratamento e recuperação, além da assistência à mulher em ginecologia, no pré-natal, parto e puerpério, no climatério, em planejamento familiar, e ainda a identificação de Doenças Sexualmente Transmissíveis (DST), câncer de colo de útero e de mama (BRASIL, 2013). Em 2000, surge o Programa de Humanização ao Pré-natal e Nascimento (PHPN), tendo em vista o conceito de humanização e qualidade na assistência à gestante durante o pré-natal, parto e puerpério e ao recém-nascido, e a redução das taxas de morbimortalidade materna e perinatal (BRASIL, 2011).

Em 2004, o MS iniciou a implementação da Política Nacional de Atenção Integral à Saúde da Mulher (PNAISM), constituída por questões de gênero reprodutivo e sexuais, violência doméstica e sexual, e saúde mental, considerando os diversos segmentos sociais na redução da morbimortalidade (BRASIL, 2012). Em 2011, é implantado pelo MS a Rede Cegonha, para melhoria dos indicadores de mortalidade, uma rede de cuidados que asseguram à mulher o direito ao planejamento reprodutivo e a atenção humanizada no prénatal, parto e puerpério, e o direito ao nascimento seguro, ao crescimento e desenvolvimento saudável da criança (BRASIL, 2011). Dessa maneira, as ações de ampliação e melhoria do planejamento reprodutivo e do pré-natal são desempenhadas com foco na atenção a mulher e a criança durante a gravidez, parto/nascimento e puerpério (LEAL MC, et al., 2019).

A Rede Cegonha por meio do SUS oferece assistência de planejamento familiar, pré-natal, parto, pósparto, e os dois primeiros anos de vida da criança. Dentre os recursos oferecidos, a rede dispõe de exames de pré-natal, de teste rápido de gravidez e de detecção da sífilis e HIV. Ainda a ampliação e qualificação de leitos de Unidade de Tratamento Intensivo (UTI) adultos e UTI neonatal, leitos de gestação de alto risco, bem como a adequação das maternidades e a construção de Centros de Parto Normal, conforme critérios estabelecidos pelas portarias do MS (BRASIL, 2013). A rede cegonha promove cursos de residência e especialização nas áreas da saúde da mulher e da criança, especificamente para a enfermagem obstétrica, e ainda formação de profissionais para atuar nos núcleos hospitalares e comitês de vigilância do óbito materno, infantil e fetal (LEAL MC, et al., 2019). 
Devido ao acúmulo de metodologias, experiências, conhecimento e a necessidade de formação de profissionais. Surgiu a necessidade de formulação do projeto Apice On (Aprimoramento e Inovação no Cuidado e Ensino em Obstetrícia e Neonatologia). Uma estratégia de indução e articulação de ações promovidas para qualificação de serviços, tendo como foco hospitais com atividades de ensino, fazendo-se as melhores práticas de atenção e cuidado ao parto e nascimento, planejamento reprodutivo, atenção às mulheres em situações de violência sexual e de aborto, no ano de 2016, participaram 96 instituições de saúde, nos quais 14 hospitais na Região Norte, 19 no Sul, 35 no Sudeste, 11 no Centro-Oeste e 17 hospitais no Nordeste (BRASIL, 2017; MARQUES GM, et al., 2019).

\section{Categoria II: As características dos óbitos maternos.}

Nos últimos anos, a mortalidade materna no Brasil vem sendo amplamente documentada. As principais causas da mortalidade materna, estão diretamente relacionadas as causas diretas ou indiretas, que demonstram ser um agravante na expectativa de vida da mulher (SCHOLZE AR, et al., 2020).

Dias JMG, et al. (2015), relata que no Brasil $80 \%$ dos óbitos maternos são decorrentes de causas obstétricas diretas, por outro lado, $20 \%$ são responsáveis por causas obstétricas indiretas. Vale ressaltar que os óbitos maternos diretos são os mais evitáveis, já que podem ser diagnosticadas e controladas no pré-natal, por exemplo: A hemorragia que está relacionada ao descolamento prévio de placenta e a problema circulatório, sendo que a eclampsia está relacionada à pressão alta e ao diabetes.

De acordo com Fonseca MCC (2019), após a realização de um levantamento no Sistema de Informações sobre Mortalidade no Departamento de Informática do Sistema Único de Saúde (DATASUS), as principais causas de óbitos maternos no Brasil, no período entre 2010 a 2017, observou-se que as três principais causas que merecem atenção e que apresentaram os maiores índices, foram a hipertensão, hemorragia e doenças do aparelho circulatório. Em um estudo realizado por Soares FAF (2017), relata que as principais causas de óbitos, entre elas podem ser citas as hipertensivas e hemorrágicas, as quais mais prevaleceram em sua revisão. Fonseca MCC (2019), corrobora que no contexto brasileiro, as causas hipertensivas estão no topo da lista, seguida das hemorrágicas e infecções.

O óbito materno tornou-se um dos grandes desafios da saúde pública no Brasil, pois suas causas não dependendo somente das condições de saúde maternas, e sim de múltiplos fatores como as características: demográficos, econômicos, políticos e sociais, que interligados, contribuem para a ocorrência de óbitos maternos (SOARES FAF, et al., 2017).

Estudos realizados no Brasil em 2015, que resultou em uma Razão de Mortalidade Materna (RMM) de 57,6 óbitos a cada 100 mil nascidos vivo observou as características sociodemográficas de mulheres que morreram por causas maternas. Em seu estudo observou-se que a maior parte das mortes maternas representavam mulheres de 20 a 34 anos, de 8 a 11 anos de estudo, solteiras, pardas e dona de casa (FEITOSA-ASSIS AI e SANTANA VS, 2020). Andrade JA (2019) relatou as características sociodemográficas de mulheres que morreram por causas maternas, entre os anos de 2007 a 2016 no município de Manaus no Amazonas. Em seu estudo observou-se que a maior parte das mortes maternas representavam mulheres de 20 a 39 anos, com 8 a 11 anos de estudo, solteiras, pardas e dona de casa, e com os índices superiores quando comparado com a média nacional.

No Brasil entre os anos de 2000 a 2009, com referência ao estado civil, a mortalidade materna foi mais elevada entre as mulheres classificadas como solteiras. O estudo foi realizado por meio do DATASUS e do Instituto Brasileiro de Geografia e Estatística (IBGE), o estudo relata que as gestantes solteiras, assim como as viúvas e separadas, tendem a ser mais vulneráveis, pela falta de suporte afetivo, emocional, social e financeiro (SCARTON J, et al., 2019). Em um estudo realizado por Leite SM (2019), sobre mortalidade materna segundo os dados sociodemográficos no Brasil, entre 2007 a 2017, observou-se em relação ao nível, escolaridade de mulheres com 8 a 11 anos de estudo, uma prevalência de $31,2 \%$ de morte materna, o que contradiz o esperado com o resultado obtido, pois quanto maior o tempo de estudo da mulher, mais orientada estaria para buscar os serviços de saúde, o que diminuiria a incidência de complicações, e consequentemente reduzindo a morte das mulheres. 
Para Vicente AC, et al. (2017), quanto maior o grau de escolaridade, maior é a preferência das mulheres por parto cesáreo, que quando realizado sem as devidas indicações estão associadas a maiores riscos para a saúde como: complicações anestésicas, hemorragias, infecções puerperais, embolia pulmonar, que podem levar a morte materna. Feitosa-Assis Al e Santana VS (2020), relataram em seus estudos que as empregadas domésticas apresentaram risco de morte materna quase quatro vezes maior que o grupo de referência. As condições de trabalho são conhecidas por extensas jornadas, comumente acima de oito horas diárias e mais de cinco dias por semana e com baixos salários. O MS relata que as características sociodemográficas, baixo grau de escolaridade, as condições socioeconômicas precárias, podem contribuir para os elevados índices de mortalidades maternas do país (BRASIL, 2012).

\section{Categoria III: Condutas para a prevenção de mortalidade em mulheres.}

Para reduzir o índice de óbito materno no âmbito hospitalar, algumas estratégias são necessárias, como na educação continuada com a oferta de cursos para aprimorar o conhecimento dos profissionais, o uso racional e planejado dos recursos, aumentar o número de leitos obstétricos e insumos. Essas estratégias podem contribuir na redução das mortes maternas, junto a campanhas estaduais para informar as mulheres sobre a importância do pré-natal e sobre os sinais de alerta para a gestante procurar o serviço de saúde (LEITE SM, 2019; LUZ MGK, et al., 2018). A qualificação dos profissionais da área da saúde, com equipes de multiprofissionais treinados para a atenção ao parto, pós-parto imediato, busca à efetividade e segurança das práticas ofertada, esses serviços de atenção obstétrica e neonatal devem contar com uma ambiência adequada, que toma o cuidado de forma horizontal, e que busca classificar o risco no momento do acolhimento (XAVIER FMSPS, 2016).

Figueiredo KMS, et al. (2018) relatam que a interdisciplinaridade entre os profissionais facilita 0 atendimento integrado à gestante. A capacidade de atuação em equipe permite aos profissionais interagir $\mathrm{e}$ desenvolver planos de cuidados que promovam a integração dos profissionais que atuam na atenção primária à saúde da mulher para a redução do índice de mortalidade materna. Na formação continuada dos profissionais da saúde destaca-se a formação de obstetrizes e/ou enfermeiras obstetras habilitadas que promovem atenção qualificada. A capacitação e atualização teórica e clínica desses profissionais estão associadas a menores taxas de intervenções. O papel do profissional de enfermagem obstétrica na prevenção da mortalidade materna é considerado fator importante na redução dos elevados índices de morte materna do país (CUNHA MA, et al., 2012).

O papel do enfermeiro, que é um profissional capaz de fortalecer as ações realizadas durante o seguimento das consultas, e tem importância fundamental na assistência pré-natal, visando à promoção da saúde e contribuindo para a garantia dos direitos das mulheres. Portanto, faz-se necessária atuação do profissional na assistência à saúde da mulher, já que a importância da enfermagem na prevenção da mortalidade materna é reconhecida na portaria do Ministério da Saúde (ARAÚJO DMR, et al., 2010; LEITE SM, 2019).A atenção à mulher no período pós-parto imediato nas primeiras semanas é imprescindível pelo menos uma visita ambulatorial para a detecção de intercorrências próprias do período puerperal. Com realização de uma anamnese detalhada e exame físico completo da puérpera. É importante o profissional da saúde verificar o cartão da gestante e avaliar o resumo da alta hospitalar, indagando a mulher sobre o parto, se houve alguma irregularidade no parto ou pós-parto (convulsões, hemorragia, febre e/ou hipertensão) (BRASIL, 2012; LEITE SM, 2019).

Ao estabelecer o princípio da equidade associado à assistência obstétrica adequada, podem contribuir significativamente à saúde da mulher. A implementação de algumas medidas de ações durante o pré-natal pode prevenir diversas complicações e eventuais óbitos maternos. As ações tomadas incluem: identificar, avaliar e tratar os fatores de risco no início do pré-natal e encaminhar as mulheres aos serviços especializados no prazo adequado e garantir a continuidade do cuidado (PACAGNELLA RC, et al., 2018). Correia RA, et al. (2011) indica que há uma necessidade de um bom acompanhamento pré-natal, com realização de consultas e exames adequados, para um maior conhecimento do desenvolvimento da gestação, procurando diminuir os riscos maternos associados a gestação, dando uma atenção ao parto e pós-parto de qualidade.

A hemorragia obstétrica é uma das principais causas de mortes maternas evitáveis no mundo. Portanto, uma das estratégias usada para reduzir a mortalidade materna foi a introdução de novas tecnologias de baixo 
custo para o cuidado da hemorragia pós-parto, tais como: balões hemostáticos intrauterinos, vestimentas antichoque não pneumáticas e "kits" de ferramentas para hemorragia obstétrica. Isso ajuda os profissionais de saúde a desenvolver processos de trabalho em suas instalações para o reconhecimento oportuno e respostas bem organizadas de situações de hemorragia obstétrica (OSANAN GC, et al., 2018).

A investigação dos óbitos maternos proporciona informações sobre os problemas obstétricos que servem de guia para o desenvolvimento de intervenções voltadas para prevenção desse tipo de morte no futuro. A mortalidade materna ainda é um grande desafio a ser combatida, e algumas condutas são necessárias para a redução da mortalidade como: a otimização a assistência hospitalar na rede básica de saúde, a educação continuada dos profissionais de saúde e o aprimoramento no diagnóstico precoce no tratamento relacionado ao período gravídico-puerperal de forma direta ou indireta (LUZ MGK, et al., 2018).

\section{CONSIDERAÇÕES FINAIS}

A mortalidade materna ainda é um problema de saúde pública no Brasil, neste sentido, verificou-se que as características das mortes maternas, no momento do óbito, aconteceram durante o puerpério, até 42 dias. O presente estudo mostra que às três principais causas diretas e indiretas que merecem atenção são a hipertensão, a hemorragia e doenças do aparelho circulatório e que apresentaram os maiores índices de óbitos maternos no Brasil, estando diretamente relacionados com os fatores sociodemográficos. Condutas devem ser iniciadas para minimizar esta problemática, como a implantação de intervenções efetivas da equipe multiprofissional, um bom acompanhamento pré-natal, realização de consultas e exames adequados, procurando diminuir os riscos maternos associados a gestação, dando uma atenção ao parto e pós-parto de qualidade, sendo relevante a capacitação dos profissionais de saúde.

\section{REFERÊNCIAS}

1. ARAÚJO DMR, et al. Depressão no período gestacional e baixo peso ao nascer: uma revisão sistemática da literatura. Cad. Saúde Pública, 2010; 26(2): 219-27.

2. CORREIA RA, et al. Características epidemiológicas dos óbitos maternos ocorridos em Recife, PE, Brasil (2000-2006). Rev. bras. enferm, 2011; 64(1): 91-97.

3. CUNHA MA, et al. Assistência pré-natal por profissionais de enfermagem no município de rio Branco, Acre, Amazônia. Revista Baiana de Saúde Pública, 2012; 36(1): 174-190.

4. DIAS JMG, et al. Mortalidade materna. Rev Méd. Minas Gerais, 2015; 25(2): 174-75.

5. FERRAZ L e BORDIGNON M. Mortalidade materna no Brasil: uma realidade que precisa melhorar. Rev Baiana Saúde, 2012; 36(2): 527-38.

6. FERREIRA LO. A emergência da medicina tradicional indígena no campo das políticas públicas. Hist. Cienc. SaudeManguinhos, 2020; 20(1): 203-219.

7. FIGUEIREDO KMS, et al. Actions of primary health care professionals to reduce maternal mortality in the Brazilian Northeast. Int J Equity Health, 2018; 17(104): 1475-9276.

8. GONÇALVES JMD, et al. Mortalidade materna. Rev Med Minas Gerais, 2015; 25(2): 173-179.

9. GUIMARÃES TA, et al. Mortalidade materna no brasil entre 2009 e 2013. Rev. Pesq. Saúde, 2017; 18(2): $81-85$.

10. KALE PL, et al. Mortes de mulheres internadas para parto e por aborto e de seus conceptos em maternidades públicas. Ciênc. Saúde Coletiva, Rio de Janeiro, 2018; 23(5): 1577-90.

11. LEAL MC, et al. Avanços na assistência ao parto no Brasil: resultados preliminares de dois estudos avaliativos. Cad. Saúde Pública, Rio de Janeiro, 2019; 35(7): e00223018.

12. LUZ MGQ, et al. Estratégias de combate ao óbito materno no âmbito hospitalar. Res. Med. J, 2018; 1(4):e39.

13. MARQUES GM, et al. Estratégias de melhoria na atenção ao parto no Brasil. Ciênc. Saúde Coletiva, Rio de Janeiro, 2019 24(2): 1678-4561.

14. MEDEIROS LT. Mortalidade materna no estado do amazonas: Estudo epidemiológico. Rev. baiana enferm. $2018 ; 32: 26623$.

15. OSANAN GC, et al. Estratégia para Zero Mortes Maternas por Hemorragia no Brasil: Uma Iniciativa Multidisciplinar de Combate à Morbimortalidade Materna. Rev. Bras. Ginecol. Obstet., Rio de Janeiro, 2018; 40(3): 103-105.

16. PACAGNELLA RC, et al. Maternal Mortality in Brazil: Proposals and Strategies for its Reduction. Rev. Bras. Ginecol. Obstet., Rio de Janeiro, 2018; 40(9): 501-506.

17. SANTOS DR, et al. Mortalidade materna na população indígena e não indígena no Pará: contribuição para a vigilância de óbitos. Esc. Anna Nery, Rio de Janeiro, 2017; 21(4): e20170161.

18. SCARTON J, et al. Perfil de mortalidade materna: uma revisão integrativa da literatura. Cuidado e Fundamental. Minas Gerais, 2019; $11(3): 816-822$.

19. SERRUYA SJ, et al. O Programa de Humanização no Pré-natal e Nascimento do Ministério da Saúde no Brasil: resultados iniciais. Cad. Saúde Pública, Rio de Janeiro, 2004; 20(5):1281-1289.

20. SOARES FAF, et al. Óbito materno, causalidade e estratégias de vigilância: uma revisão integrativa. Revista Eletrônica Acervo Saúde, 2017; 9: S890-S897.

21. VIANA RC, et al. Mortalidade Materna: uma abordagem atualizada. Comunicação em Ciências da Saúde, 2011; 22(1): 141152.

22. VICENTE AC, et al. Parto cesáreo e parto normal: uma abordagem acerca de riscos e benefícios. Temas em Sáude, João Pessoa, 2017; 17(4): 24-35.

23. SCHOLZE AR, et al. Mortalidade materna: comparativo após implantação da Rede Mãe Paranaense. J. Nurs. Health., 2020; 10(2): e20102007. 